\title{
Ultrasonography in the diagnosis and management of non inflammatory conditions of the hand and wrist
}

\author{
Florentin Ananu Vreju ${ }^{1 *}$, Marius Eugen Ciurea ${ }^{2 *}$, Dragos Popa², Florin Popa ${ }^{3}$, \\ Cristina Dorina Parvanescu ${ }^{4}$, Beatrice Andreea Chisalau ${ }^{4}$, Andreea Lili Barbulescu ${ }^{4}$, \\ Vlad Parvanescu², Anca Rosu' ${ }^{1}$, Paulina Lucia Ciurea ${ }^{1}$
}

\begin{abstract}
${ }^{1}$ Rheumatology Department, University of Medicine and Pharmacy, ${ }^{2}$ Plastic Surgery Department, University of Medicine and Pharmacy, ${ }^{3}$ Plastic Surgery Department, Emergency County Hospital, ${ }^{4}$ Rheumatology Department Emergency County Hospital, Craiova, Romania

* the authors shared the first authorship
\end{abstract}

\begin{abstract}
High resolution ultrasonography has already become an important tool in the diagnosis and management of inflammatory arthritis of the hand and wrist, but lately it has been proven to be the method of choice in the evaluation of traumatic lesions of tendons, annular pulleys, nerves, and ligaments and at the same time in detecting foreign bodies. The objective of this paper is to review and describe the current knowledge on these US findings and to highlight the ultrasonography role in the evaluation of non-inflammatory conditions of the hand and wrist.
\end{abstract}

Keywords: tendon, nerves, ultrasonography, injury, wrist, hand surgery

\section{Introduction}

The hand and wrist are the most used parts of the body during a human's daily life, especially for individuals with manual jobs, and this leads frequently to traumatic lesions of the bone, joints, nerves and tendons. Sport or work injuries of the hand and wrist constitute up to $28 \%$ of all musculoskeletal injuries [1,2]. In a study on 50,272 injured patients, up to $30 \%$ of the patients that presented in the emergency room, had trauma of the hand and wrist [3].

Due to the improvement of imaging techniques in the last years, the performance of ultrasonography (US) in detecting pathological changes in the musculoskeletal system dramatically increased. Moreover, dynamic examination allows better evaluation of the type and exten-

Received 15.11.2015 Accepted 02.12.2015

Med Ultrason

2016, Vol. 18, No 1, 90-95

Corresponding author: Florentin Ananu Vreju

Rheumatology Department

University of Medicine and Pharmacy Craiova

Petru Rares 2-4, Craiova, Romania

E-mail: florin_vreju@yahoo.com sion of the lesions. An anatomical course of the nerves and tendons can be depicted by US, thus offering valuable information on the exact location and type of possible lesions [4].

The aim of this paper is to review the current literature data regarding non-inflammatory conditions of the hand and wrist and to highlight the role of US in their evaluation.

\section{US examination}

Musculoskeletal US of the hand and wrist can depict subtle details of the nerves, tendons, or ligaments, if a proper examination technique is used. Besides this, knowledge of relevant anatomy and artefacts is essential for an accurate diagnosis [4]. As equipment, high frequency linear-array transducers of $10-18 \mathrm{MHz}$, are mandatory for the correct evaluation of the hand and wrists structures. The patients usually are seated with hands placed on a table, to permit examination of both volar and dorsal aspects, in longitudinal and transversal axis. Examination of the hands and fingers usually requires a large amount of transmission gel, in order to avoid com- 
pression and to permit dynamic evaluation [5]. When acute trauma with skin lesions is investigated, sterile transmission gel, or sterile acoustic standoff pads can be used.

Commonly, the correct evaluation of the wrist tendons starts in the transverse plane (short axis), because of the high number of tendons. After recognizing the tendon or compartment in short axis, the structure is evaluated in the longitudinal plane in order to confirm the type and accurate location of the lesion.

\section{Bones injuries (fractures)}

In patients treated for hand injuries the fractures are the most frequent lesions $(42 \%)$, followed by the tendons $(29 \%)$ and skin lesions, as showed in the study of Schoffl et al [3]. Even if fractures diagnosis remains mainly based on conventional radiography (CR), computer tomography $(\mathrm{CT})$, or magnetic resonance imaging (MRI), US is a highly accurate method for the evaluation of the bone surface, being able to detect even small, occult irregularities of the bone cortex. US has a high sensitivity and specificity in detecting fractures, especially in evaluating long bones linear diaphysis and metaphyseal region, close to the skin. Bone cortical appears as a bright hyperechoic smooth line, with acoustic posterior shadowing and possible reverberation artefact [6]. Fractures appear as the interruption of the hyperechoic line of the bone cortical, with hypoechoic periosteal thickening and surrounding soft tissue oedema (fig 1). Increased hyperaemia at the fracture's site is possible. If cortical irregularity can be easily seen in the dyaphysis of long bones, in epiphyseal bone or irregular ones the

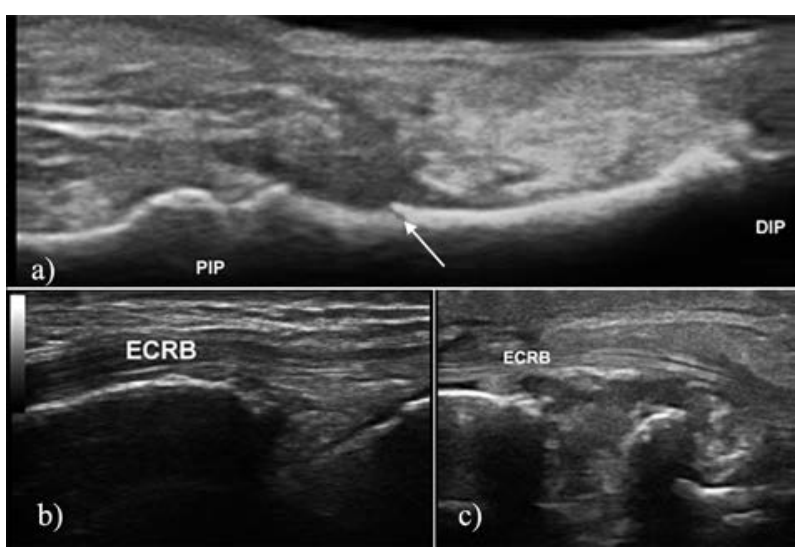

Fig 1. a) Intermediate phalanx fracture (arrow), volar aspect. PIP - proximal interphalangeal joint, DIP - distal interphalangeal joint; b) Normal appearance of the radioscaphoid joint; c) Radioscaphoid joint, with fracture of the scaphoid and secondary hypoechoic collection surrounding, dorsal aspect. ECRB extensor carpi radialis brevis tendon.
US beam needs to be oblique, which may result in the loss of details [7].

The US value in the diagnosis of irregular bone fractures, especially in scaphoid, was previously discussed [8-12], with the conclusion that US might be valuable even in patients without changes in CR. At the same time, other studies showed that US can depict more fractures than CR [13-15] and should be considered an alternative for the more expensive and less available MRI and CT. In a recent published case report, fractures secondary to hyperextension of the thumb, of both the ulnar and radial sesamoids of the metacarpophalangeal joint of the thumb were diagnosed by US and confirmed by CT. When an irregular bone fracture is suspected and CR is negative, MRI or CT scan can be used to confirm US results [16].

Furthermore, US can be used to identify new bone formation, before being visible on $\mathrm{CR}$, as echogenic foci at the fracture site, which become aligned in long axis [17], while fluid in the distraction gap might suggest a low rate of new bone formation.

Dynamic evaluation is another advantage of US, besides the availability and multiplanar capability, which can demonstrate a fracture non-union during active or passive motion.

\section{Tendons and annular pulleys lesions}

The second most frequent injuries in the hand involves the tendons. The recognition of a full tendon rupture is usually simple, based on history and physical examination: an acute pain, associated with a popping sound (described by the patient during injury), followed by a special position of the finger [5]. Still, in complicated cases, with more than one tendon involvement, in postsurgical evaluation of tendon repair, or in severe wounds, with more than one structure involved, imaging techniques are essential for diagnosis.

Tendons have a specific fibrillar echotexture, due to the parallel collagen fibers arranged into bundles, and are easily depicted by experienced ultrasonographers. At the same time, it is worth mentioning that tendons express clearly one of the most frequent artefacts - anisotropy, which translates into a reduction in the echogenicity, due to the oblique angle of the US beam with the fibers and can lead to misinterpretation. Höglund et al described in 1991 the utility of US, in the detection of complete tears in recently repaired tendons [18] and later, Corduff et al followed the healing process of the repaired flexor tendons using US [19].

The type of the tendon injury is important for the patient outcome, treatment options, and for monitoring the healing process. The injury patterns are differentiated 
into injuries of the main tendon, of a tractus or pulley, or of the tendon insertion, as well as injury of the dorsal or volar tendons. US can help identify the types and the severity of the lesions and suggest the most accurate individual management. For example, lesions of less than $60 \%$ of the tendon's diameter should be treated conservatively, as the outcome is better, with a tear-resistance higher than in surgically treated tears [20].

Post-injury, the healing process could be extrinsic, such as starting from the fibroblasts in peritendinous tissues, with migration and adhesion, or intrinsic healing, supported by immigration of fibroblast-like tenocytes, secondary to early passive movement [3].

Typical US findings in an injured tendon are a hypoechoic area within the tendon, interruption of longitudinal fibrilar aspect, tendon swelling, or even a gap inside the tendon in the case of complete rupture $[21,22]$. The difference between a recent and sub-acute/chronic total tear can be made by the aspect of the gap between the two ends of the tendon: if it is filled with anechoic fluid, the injury is recent, while hyperechoic or mixed material, suggesting fibrinous tissue, can be revealed in a late presentation.

Complex injuries, with involvement of the surrounding tissues, might present without tendon gaps, with an isoechoic aspect of the lesion compared to the rest of the tendon. Sometimes loss of the fibrilar aspect of the ten-

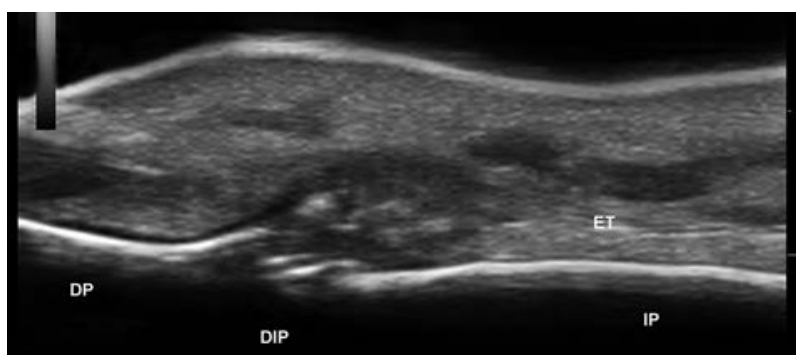

Fig 2. Avulsion fracture in the mallet finger - hyperechoic bone fragment superficial to DIP. IP - intermediate phalanx, DP distal phalanx, ET - extensor tendon, DIP - distal interphalangeal joint

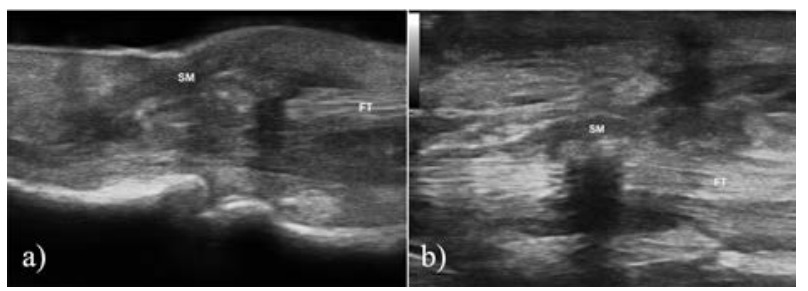

Fig 3. Repaired tendons, with hyperechoic area and posterior shadowing due to suture material: a) longitudinal scan of PIP joint, volar aspect, with flexor tendon sutured; b) longitudinal scan of the wrist, volar aspect, with the 2nd finger superficial flexor tendon sutured. don is possible. In these situations, dynamic US examination might reveal no gliding of the tendon during finger movements, due to adherence to other soft tissues [5].

Boutonniere deformity, defined as lesion in the central strip of the extensor tendons at the level of the PIP joint, with slips of both lateral tendon strips, might be seen in US as an interruption of the tractus intermedius.

Avulsion fractures are commonly found at the level of the deep flexor tendon insertion on the distal phalanx. These types of lesions are more frequent in the $4^{\text {th }}$ finger and appear after forced extension of the digit [3]. When extensor tendon is involved, at the level of the distal interphalangeal joint, one third of the patients might present with mallet fractures (fig 2). In US, we can depict the avulsion fractures as hyperechoic areas at the end of the tendons, surrounded by hypoechoic areas due to local oedema and inflammation.

Repaired tendons might have different aspects in US, from hypoechoic to hyperechoic, sometimes with loss of the fibrilar pattern, due to varying degrees of granulation tissue. In postsurgical tendons hyperechoic suture material, with posterior acoustic shadowing or reverberation artefacts can be observed as the suture catgut is frequently a non-resorbable material (fig 3 ). Thus, the normal criteria for tendon tear, such as hypoechoic area or tendon thinning, might not be applied to postoperative tendon, as the hypoechoic defects, within repair area, might persist for years [23]. This could lead to the misinterpretation of the severity of the lesion. In all these cases, dynamic examination could clarify the situation.

Chronic microtrauma at the level of the extensor retinaculum in the first compartment of extensor tendons of the wrist might lead to de Quervain disease, a tendinosis of the extensor pollicis brevis and adductor pollicis longus tendons, with thickening of the retinaculum. By US, de Quervain disease is identified as the swelling of the first compartment of extensors, with a hypoechoic aspect of the area surrounding tendons. Fluid can be observed in acute lesions in the tendon sheet and even proliferated and vascularizated synovium [7]

Injuries of the pulleys are frequently associated with flexor tendons lesions, as they form a functional unit. Using US the diagnosis of a pulley lesion is revealed during the examination with the finger in forced flexion and pressure towards the probe, permitting the evaluation of the distance between the tendon and the bone cortex [24,25]. If the distance is $\leq 2 \mathrm{~mm}$ or higher, but a single pulley ruptures, the patient should benefit from conservative therapy. Otherwise, surgical repair is recommended [23]. Klauser et al [26] suggested that a distance of $\geq 3$ $\mathrm{mm}$ at rest, or $5 \mathrm{~mm}$ during movement, can confirm a pulley rupture. 


\section{Nerves}

US examination of the nerves was used for the first time to study recurrent laryngeal nerve aspect [27] in 1985 , by Solbiati, followed by Fornage in 1988, who evaluated peripheral nerves in healthy subjects [28]. Silvestri et al described the normal structure of the nerve both in longitudinal and transverse sections. In longitudinal aspect, the normal appearance of the nerve in US is hyperechoic compared to muscle and hypoechoic compared to tendon. It is important to depict between the "fibrilar echotexture" of the tendons and the "fascicular echotexture" of the nerve [29], with multiple hypoechoic parallel linear areas, corresponding to fascicles of neuronal fibers, separated by hyperechoic perineurium [4]. On transverse section, the nerve appears as an oval shaped area, with round hypoechoic spots on the hyperechoic background, giving the "salt and pepper" aspect.

The architecture, shape and continuity of the nerves are evaluated in both perpendicular transverse and longitudinal planes.

Nerve lesions are commonly diagnosed by clinical and electrophysiological examination, but these methods are not accurate on the location and severity of the lesion. Electrophysiological examination might offer some information on the location of the nerve injury, but only after nerve repair, which occurs commonly after 6-8 weeks post-injury. In the acute phase, US might offer more information on the full or partial nerve lesion, or on the presence of foreign bodies or hematoma [30]

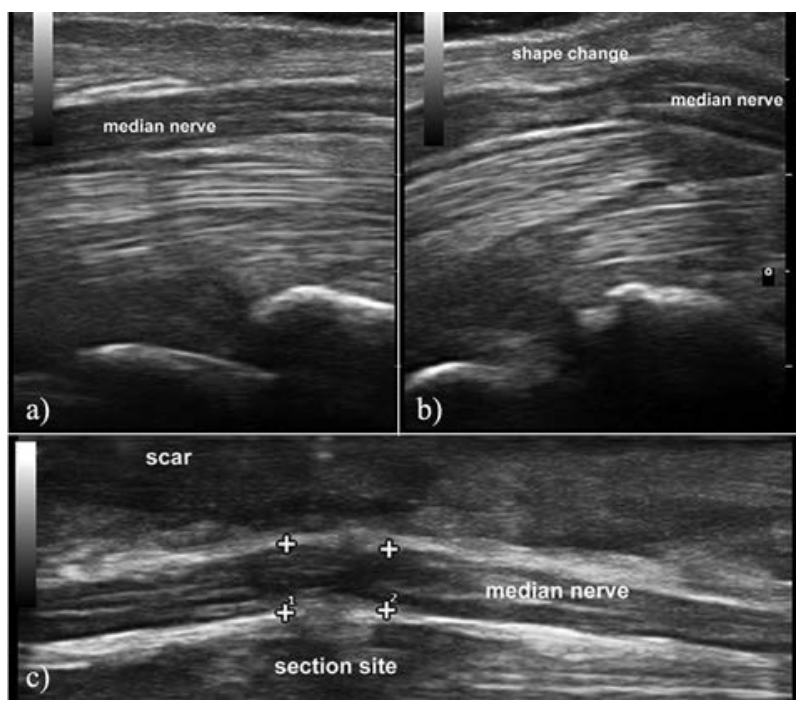

Fig 4. a) Longitudinal scan of the wrist, volar section, with normal median nerve; b) median nerve with change in shape, secondary to callus on the lunate bone; c) longitudinal scan of the wrist, volar section, with a sutured median nerve, secondary to injury. and thus facilitating diagnosis and helping in the optimal choice of treatment. Bodner et al demonstrated in a small study that acute nerve damage or chronic impairment by posttraumatic tissue such as callus, bone fragments or scars can be identified by US [31] (fig 4). In the chronic phase, neuromas could be visualized as a well defined hypoechoic mass [32].

\section{Ligaments}

Wrist ligaments injuries, including those in the scapholunate or lunotriquetral, might be visible by US examination. In the hand, US is able to identify the finger and thumb collateral ligaments of the metacarpophalangeal (MCP) and interphalangeal joints. Injuries of the ulnar (UCL) and radial (RCL) collateral ligaments of the thumb are common in sports. Most frequently, lesion of the UCL appears especially while skiing ("skier's thumb").

Normal UCL is hyperechoic and can be identified on the ulnar side of the MCP joint, profound to a thin hyperechoic band which corresponds to adductor pollicis aponeurosis.

Spectrum of UCL injuries of the thumb include: strain, partial thickness tear, full thickness tear, and associated findings like bone avulsion, volar plate injury, or Stener lesion (full thickness tear of the UCL, with significant retraction of the proximal part and its positioning over superficial to adductor pollicis aponeurosis) [7]. It is essential to diagnose specific degrees, from strain to Stener lesion, or bony lesions, as these might make the management more difficult $[7,33]$. US may help differentiate between the 6 types of skier's thumb and indicate surgical or conservatory treatment, with splints or casts [33].

Lesion of the RCL are less common but due to the intrinsic anatomy of the radial side of the first MCP joint, combined with the dynamic force of adductor pollicis tendon, those lesions are usually leading to a rapid pathologic process, with joint instability and progressive volar subluxation. US appearance is similar to UCL lesions, apart from the changes related to Stener lesions [7].

Considered a ligament, the volar plate is another structure frequently involved in sport injuries. In US, it appears as a triangular echogenic structure deep in the flexor tendons, displacing it from the bony surface. Volar plate injuries are the result of forced hyperextension in the MCP joint and might be seen in US as a mixed hypo-hyperechoic irregular area involving the echogenic structure of the volar plate [7,34]. If the injury is associated with a characteristic phalangeal avulsion fracture, the fragment can be identified by US as a hyperechoic image, with acoustic shadow. 


\section{Foreign bodies}

US is an excellent imaging method to identify possible foreign bodies. Frequently, foreign bodies appear as hyperechoic structures (fig 5) with acoustic shadowing or a comet tail artefact in the case of metal. Wood and glass have a lower reflectivity compared to metal. In chronic lesions, a hypoechoic area surrounding the foreign body might be seen, due to inflammation or granuloma formation. US evaluation, with accurate positioning of the foreign body and depictions of the surrounding anatomical structures, might improve the outcome, by optimizing surgical procedure and minimizing damage [5].

\section{Other lesions}

US can depict collections in infections secondary to trauma, which appear as hypoechoic/anechoic areas ill defined, with Doppler signal in the surrounding tissues (fig 6). In these cases, US can help decide the need for surgical or conservatory treatment and at the same time, can offer information on the structures involved.

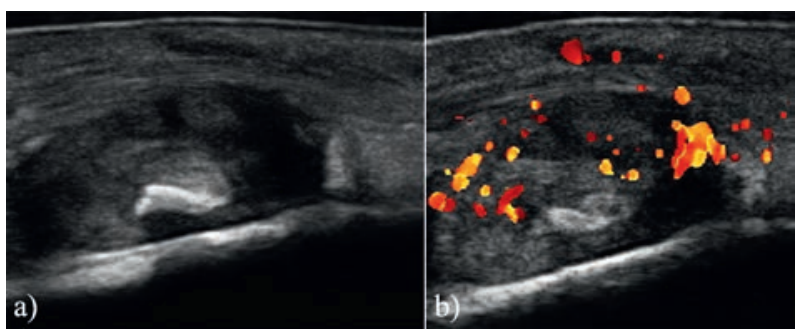

Fig 5. Longitudinal $(a, b)$ and transverse (c) scans at the level of metacarpal bones, showing hyperechoic foreign rubber body, with hypoechoic area surrounding it, due to local inflammation.

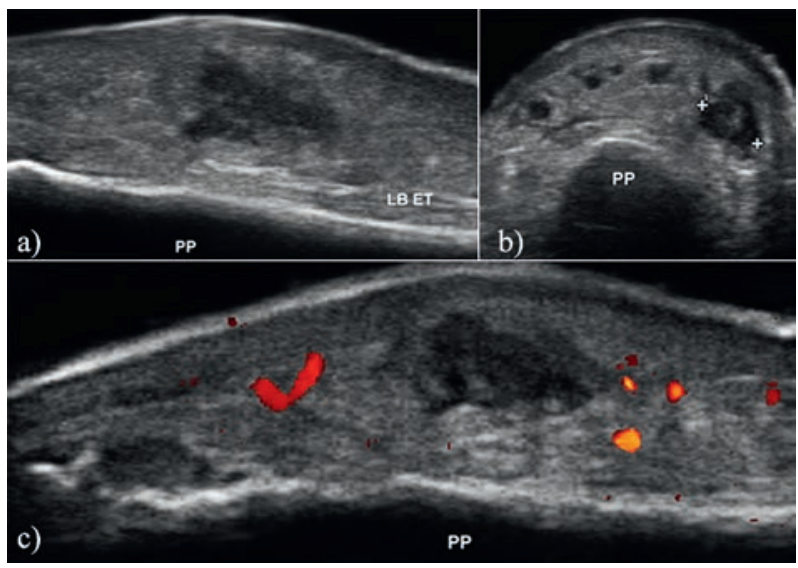

Fig 6. Longitudinal $(a, c)$ and transverse scans (b) at the level of proximal phalanx, showing hypoechoic collection area, with PD signal, close to the lateral band of the extensor tendon. PP proximal phalanx, LB ET - lateral band of the extensor tendon

\section{Conclusions}

Non-inflammatory conditions of hand and wrist are common and US, as a noninvasive, cost-effective accurate diagnostic tool, could play an important role in the imaging of hand and wrist pathology. In conclusion, the benefits of US described above provide important support for physicians in their daily practice, shortening the time to treatment and optimizing management of noninflammatory hand and wrists conditions.

Acknowledgement: This paper was published under the frame of the European Social Found, Human Resources Development Programme 2007-2013, POSDRU/159/1.5/S/136893.

\section{Conflict of interest: none}

\section{References}

1. Trybus M, Lorkowski J, Brongel L, Hladki W. Causes and consequences of hand injuries. Am J Surg 2006; 192: 5257.

2. Angermann $P$, Lohmann M. Injuries to the hand and wrist. A study of 50,272 injuries. J Hand Surg Br 1993; 18: 642644.

3. Schoffl V, Heid A, Kupper T. Tendon injuries of the hand. World J Orthop 2012; 3: 62-69.

4. Koenig RW, Pedro MT, Heinen CP, et al. High-resolution ultrasonography in evaluating peripheral nerve entrapment and trauma. Neurosurg Focus 2009; 26: E13.

5. Karabay N. US findings in traumatic wrist and hand injuries. Diagn Interv Radiol 2013; 19: 320-325.

6. Hoffman DF, Adams E,; Bianchi S. Ultrasonography of fractures in sports medicine. Br J Sports Med 2015; 49: 152-160.

7. Ebrahim FS, De Maeseneer M, Jager T, Marcelis S, Jamadar DA, Jacobson JA. US diagnosis of UCL tears of the thumb and Stener lesions: technique, pattern-based approach, and differential diagnosis. Radiographics 2006; 26 : 1007-1020.

8. Herneth AM, Siegmeth A, Bader TR, et al. Scaphoid fractures: evaluation with high-spatial-resolution US initial results. Radiology 2001; 220: 231-235.

9. DaCruz DJ, Taylor RH, Savage B, Bodiwala GG. Ultrasound assessment of the suspected scaphoid fracture. Arch Emerg Med 1988; 5: 97-100.

10. Senall JA, Failla JM, Bouffard JA, van Holsbeeck M. Ultrasound for the early diagnosis of clinically suspected scaphoid fracture. J Hand Surg Am 2004; 29: 400-405.

11. Davis TR. Prediction of outcome of non-operative treatment of acute scaphoid waist fracture. Ann R Coll Surg Engl 2013; 95: 171-176.

12. Yildirim A, Ünlüer EE, Vandenberk N, et al. The role of bedside ultrasonography for beside ultrasound in the emer- 
gency department. Turk J Traum Emerg Surg 2013; 19: 241-245.

13. Demers G, Migliore S, Bennett DR, et al. Ultrasound evaluation of cranial and long bone fractures in a cadaver model. Mil Med 2012; 177: 836-839.

14. Chan SS. Emergency bedside ultrasound for the diagnosis of rib fractures. Am J Emerg Med 2009; 27: 617-620.

15. Turk F, Kurt AB, Saglam S. Evaluation by ultrasound of traumatic rib fractures missed by radiography. Emerg Radiol 2010; 17: 473-477.

16. Becciolini M, Bonacchi G. Fracture of the sesamoid bones of the thumb associated with volar plate injury: ultrasound diagnosis. J Ultrasound ; 18: 395-398.

17. Young JW, Kostrubiak IS, Resnik CS, Paley D. Sonographic evaluation of bone production at the distraction site in Ilizarov limb-lengthening procedures. AJR Am J Roentgenol 1990; 154: 125-128.

18. Hoglund M, Tordai P, Engkvist O. Ultrasonography for the diagnosis of soft tissue conditions in the hand. Scand J Plast Reconstr Surg Hand Surg 1991; 25: 225-231.

19. Corduff N, Jones R, Ball J. The role of ultrasound in the management of zone 1 flexor tendon injuries. J Hand Surg Br 1994; 19: 76-80.

20. Boyer MI, Strickland JW, Engles D, Sachar K, Leversedge FJ. Flexor tendon repair and rehabilitation: state of the art in 2002. Instr Course Lect 2003; 52: 137-161.

21. Jeyapalan K, Bisson MA, Dias JJ, Griffin Y, Bhatt R. The role of ultrasound in the management of flexor tendon injuries. J Hand Surg Eur Vol 2008; 33: 430-434.

22. Lee DH, Robbin ML, Galliott R, Graveman VA. Ultrasound evaluation of flexor tendon lacerations. J Hand Surg Am 2000; 25: 236-241.

23. Chun KA, Cho KH. Postoperative ultrasonography of the musculoskeletal system. Ultrasonography 2015; 34: 195205.
24. Schöffl VR, Schöffl I. Injuries to the finger flexor pulley system in rock climbers: current concepts. J Hand Surg Am 2006; 31: 647-654.

25. Klauser A, Stadlbauer KH, Frauscher F, et al. Value of transducer positions in the measurement of finger flexor tendon thickness by sonography. J Ultrasound Med 2004; 23: 331-337.

26. Klauser A, Frauscher F, Bodner G, et al. Finger pulley injuries in extreme rock climbers: depiction with dynamic US. Radiology 2002; 222: 755-761.

27. Solbiati L, De Pra L, Ierace T, Bellotti E, Derchi LE. Highresolution sonography of the recurrent laryngeal nerve: anatomic and pathologic considerations. AJR Am J Roentgenol 1985; 145: 989-993.

28. Fornage BD. Peripheral nerves of the extremities: imaging with US. Radiology 1988; 167: 179-182.

29. Silvestri E, Martinoli C, Derchi LE, Bertolotto M, Chiaramondia M, Rosenberg I. Echotexture of peripheral nerves: correlation between US and histologic findings and criteria to differentiate tendons. Radiology 1995; 197: 291-296.

30. Cokluk C, Aydin K. Ultrasound examination in the surgical treatment for upper extremity peripheral nerve injuries: part I. Turk Neurosurg2007; 17: 277-282.

31. Bodner G, Buchberger W, Schocke R, et al. Radial nerve palsy associated with humeral shaft fracture: evaluation with US-initial experience. Radiology 2001; 219: 811816.

32. Wong DC. Wansaicheong GK, Tsou IY. Ultrasonography of the hand and wrist. Singapore Med J 2009; 50: 219225.

33. Hintermann B, Holzach PJ, Schütz M, Matter P. Skier's thumb -the significance of bony injuries. Am J Sports Med 1993; 21: 800-804.

34. Anderson J, Read JW. Atlas of imaging in sports medicine. 2nd ed. McGraw-Hill, Sydney, 2007. 